\section{Re: Role of Non-Group A Streptococci in Acute Pharyngitis}

To the Editor: Jeffrey Tiemstra and Rosita L. F. Miranda ${ }^{1}$ have added important data concerning the diagnosis and management of acute pharyngitis. Like previous investigators, they found that a significant percentage of adolescents and young adults presenting with acute pharyngitis grow streptococci from a group other than group A strep, which is the classic concern.

Most other articles on this subject have focused on group $\mathrm{C}$ and group $\mathrm{G}$ streptococci. I doubt that clinical presentation of group $B$ resembles the clinical presentation of group C. In unpublished data, we did not find group B patients having such a presentation. Zwart also found that only A, C, and G caused pharyngitis. ${ }^{2}$ Therefore, I would suggest that the authors look carefully at the distribution of clinical indicators in group B compared with group A and group C.

The Tiemstra article also shows that in practice (rather than in prospective studies) the sensitivity of the group A strep rapid test was only $75 \%$. One can postulate several reasons for a difference in rapid test sensitivity from prospective studies. Just to suggest 2 possibilities: (1) sampling errors might occur more often in practice or (2) there may be publication bias for higher sensitivity studies. $^{3}$

Like Zwart's classic 2000 BMJ study on treatment, the authors find a high probability of either group A or non-group A strep in patients having high pharyngitis scores. ${ }^{3}$ That article supports a clinical improvement from antibiotics for group $C$ strep pharyngitis. In that article adults (ages 15-60) experienced a 2-day symptom improvement if they had group A pharyngitis and a 1-day symptom improvement if they had group $\mathrm{C}$ pharyngitis.

The addition of headache to the pharyngitis scores, although understandable, makes comparison of this study to previous studies more difficult.

A note of caution: these findings probably apply to the adolescent/young adult age group and not pre-adolescents. Previous studies have shown that non-group A infections occur commonly in college health populations but not pre-adolescents.

Congratulations for continuing the documentation that adolescent and adult pharyngitis is more complex than deciding whether the patient has group A strep.

Robert M. Centor, MD

University of Alabama Huntsville Regional Medical Campus, Huntsville, AL rcentor@uab.edu

\section{References}

1. Tiemstra J, Miranda RLF. Role of non-group A streptococci in acute pharyngitis. J Am Board Fam Med 2009;22: $663-9$.
2. Zwart S, Ruijs GJ, Sachs AP, van Leeuwen WJ, Gubbels JW, de Melker RA. Beta-haemolytic streptococci isolated from acute sore-throat patients: cause or coincidence? A case-control study in general practice. Scand J Infect Dis 2000;32:377-84.

3. Zwart S, Sachs AP, Ruijs GJ, Gubbels JW, Hoes AW, de Melker RA. Penicillin for acute sore throat: randomised double blind trial of seven days versus three days treatment or placebo in adults. BMJ 2000;320:150-4.

doi: 10.3122/jabfm.2010.03.090267

The above letter was referred to the author of the article in question, who offers the following reply.

\section{Response: Re: Role of Non-Group A Streptococci in Acute Pharyngitis}

To the Editor: We appreciate Dr. Centor's ${ }^{1}$ observations and comments on the literature regarding the role of non-group A strep in acute pharyngitis. In our population, group B was associated with 3 of the 4 classic criteria-fever, cervical adenopathy, and exudates-but not absence of cough (Figure 5 in our article ${ }^{2}$ ), suggesting that it may also represent a pathogen. Given the concerns for group B strep infection in the young adult population that includes pregnant women this certainly warrants further investigation, which we hope to pursue. Jeffrey Tiemstra, MD

Family Medicine Center, University of Illinois at Chicago

Chicago, IL jtiemstr@uic.edu

\section{References}

1. Centor RM. Re: role of non-group A streptococci in acute pharyngitis. J Am Board Fam Med 2010;23:423.

2. Tiemstra J, Miranda RLF. Role of non-group A streptococci in acute pharyngitis. J Am Board Fam Med 2009;22: $663-9$.

doi: 10.3122/jabfm.2010.03.100045

\section{Re: Does Having a Personal Physician Improve Quality of Care in Diabetes?}

To the Editor: We have read with interest the article by Hueston, "Does having a personal physician improve quality of care in diabetes?" in your January/February issue, but we have serious reservations about his conclusion for several reasons.

First, although he cites 4 articles coauthored by Arch Mainous, he omits the one by Mainous most directly relevant to this article, which was Mainous and Gill (1998), ${ }^{2}$ showing that for patients the benefits of continuity of care flow more from a personal relationship than from seeing others at the same site of care. 
Secondly, he examined the records of some people who had had diabetes for only 6 months, and only 2 consultations, which is much too short a period and too few contacts for effects of continuity of care mediated through a regular provider to be fairly measured.

Thirdly, he diminishes the work of O'Connor and colleagues $(1998)^{3}$ who, with a much bigger population studied, found a whole series of benefits for patients from having a regular provider. These included having better glycaemic control and receiving most recommended elements of modern disease management. His reference to those showing value in continuity as revealing some (our emphasis) benefits is inaccurate, as O'Connor and colleagues showed many important benefits.

Fourthly, having correctly reported that several studies have shown that continuity of care in family practice is associated with increased satisfaction by patients (to which we would add others ${ }^{4,5}$ ), he then ignores this very important outcome in his conclusion. There is also important evidence from Canada that continuity of care by family physicians is associated with a significantly lower rate of admissions to hospital for the elderly. ${ }^{6}$

Finally, he showed no disadvantages of continuity of care via a regular provider and did find 2 advantages, one of which, a significantly lower $\mathrm{HbA} 1 \mathrm{C}$, is the cardinal measure of diabetes control. His conclusion that there are "few benefits" of having a regular provider does not follow from his own findings.

Denis Pereira Gray, MB BChir St Leonard's Research Practice,

Devon, United Kingdom denis.pereiragray@btinternet.com

Philip Evans, MPhil

St Leonard's Research Practice, Devon, United Kingdom

Christine Wright, $\mathrm{PhD}$ St Leonard's Research Practice, Devon, United Kingdom

Peter Langley, PhD

St Leonard's Research Practice, Devon, United Kingdom

We acknowledge with thanks funding to support research infrastructure from NHS Devon.

\section{References}

1. Hueston WJ. Does having a personal physician improve quality of care in diabetes? J Am Board Fam Med 2010;23: $82-7$.

2. Mainous AG, Gill JM. The importance of continuity of care in the likelihood of future hospitalization: Is site of care equivalent to a primary clinician? Am J Public Health 1998; 88:1539-41.

3. O'Connor PJ, Desai J, Rush WA, Cherney LM, Solberg LI, Bishop DB. Is having a regular provider of diabetes care related to intensity of care and glycaemic control? J Fam Pract 1998;47:290-7.

4. Baker R, Streatfield J. What type of general practice do pa- tients prefer? Exploration of practice characteristics influencing patient satisfaction. Br J Gen Pract 1995;45:654-9.

5. Reis HT, Clark MS, Pereira Gray DJ, et al. Measuring responsiveness in the therapeutic relationship: A patient perspective. Basic Appl Soc Psych 2008;30:339-48.

6. Menec VH, Sirski M, Attawar D, Katz A. Does continuity of care with a family physician reduce hospitalizations among older adults? J Health Serv Res Policy 2006;11:196201.

doi: 10.3122/jabfm.2010.03.100034

The above letter was referred to the author of the article in question, who offers the following reply.

\section{Response: Re: Does Having a Personal Physician Improve Quality of Care in Diabetes?}

To the Editor: I appreciate the thoughtful letter from Dr. Gray and colleagues. ${ }^{1}$ The issue of continuity and its value in patient care is one that raises a great deal of passion among family physicians. The preceding letter raises some very important issues that need to be considered when continuity is examined, several of which were beyond the study recently published in the fournal. ${ }^{2}$

First, the study by Drs. Mainous and Gill ${ }^{3}$ cited by Dr. Gray and colleagues found differences in hospitalization rates based on personal continuity among Medicaid recipients in a single state. The data set used by Mainous and Gill combined patients with many different clinical conditions and, by virtue of focusing on Medicaid recipients, a population with low financial resources. In contrast, the paper on diabetes examined quality of care measures for a single disease entity when care was delivered by the same physicians in the same setting with the only difference being the extent of interpersonal continuity. In addition, physicians received continuous feedback on their performance, a factor not used in the retrospective analysis by Mainous and Gill. These differences make the current study quite different from that reported previously on data from the mid-1990s in the Mainous study.

Second, the population used in the study conducted at our center was not limited to patients with diabetes of 6 months duration. Instead, 6 months was the minimal amount of time that a patient had to have diabetes to participate in the study, and the vast majority of patients had been established for much longer than that time period. So the patients in this study did have ample time to have therapy initiated for their diabetes and related health conditions.

In the study by O'Connor and colleagues, ${ }^{4}$ it should be pointed out that there was no significant difference in mean hemoglobin $\mathrm{A} 1 \mathrm{C}$ levels between patients in the continuous care and noncontinuous care groups. O'Connor and his group observed greater proportion of patients who did not have continuity who had $\mathrm{HbA1C}$ level over $10 \%$, but this is a rather crude measurement of the effectiveness of diabetes care. All the other differences between the groups was based on self-report and 